\title{
Discrimination of Identikit constructions of faces: Evidence for a dual processing strategy
}

\author{
MICHAEL L. MATTHEWS \\ University of Guelph, Guelph, Ontario, Canada NIG 2WI
}

\begin{abstract}
Feature saliency and processing strategy in the recognition of faces are investigated in a "same"-"different" RT paradigm using pairs of faces constructed from Identikit representations. Facial similarity is varied by manipulating individual feature changes in pairs of faces. Although there was a general tendency for RT to increase as a function of facial similarity, analysis of RTs to component feature changes suggests a dual processing strategy whereby subjects give processing priority to hairline, eyes and chin, followed by a slower feature-byfeature analysis of eyebrows, nose, and mouth.
\end{abstract}

A continuing theme in the study of the perception of human faces has evolved around the issue of whether faces are processed in a Gestalt manner in which the importance of configurational relationships between features is stressed or by means of a piecemeal analysis that goes feature by feature. Variations on this central theme include the issues of serial vs. parallel processing strategies and the saliency of different facial features. The initial stimulants that produced investigations in these directions were most probably Yin's (1969) finding that subjects commonly adopted one of two strategies in attempting to recognize upside-down faces, either searching for a distinguishing feature or attempting to obtain an overall impression, and secondly, an increasing body of data from developmental psychologists on patterns of preferential fixation behavior in the scanning of representations of faces by infants.

These issues have been brought together by Bradshaw and Wallace (1971), who suggest that Gestalt or configurational encoding would be evidenced by a parallel processing strategy, whereas a serial approach would be more suggestive of separate feature processing. Using a "same"."different" RT task with lists of pairs of faces constructed from an Identikit in which pair members differed in terms of numbers of features in common, they concluded that a serial self-terminating processing model best characterized subjects' performance and that there was no evidence for faces to be treated as unitary Gestalten. This conclusion is supported by the results of Tversky (1969), who suggested that subjects per-

Requests for reprints should be sent to Michael L. Matthews, Department of Psychology, University of Guelph, Guelph, Ontario, NIG $2 \mathrm{~W} 1$. This research was supported by a grant to the author from the National Research Council of Canada. form a serial self-terminating facial search with eyes and face shape given processing priority; these results were obtained, however, with simple line-drawing schematic faces whose overall simplicity and qualitative differences from real faces, photographs of faces, or Photofit reconstructions may result in a specific task-oriented processing strategy. Using slightly more convincing face-like schematic representations, Smith and Nielsen (1970) concluded that dual processing mechanisms underlay recognition memory for faces, with "same" judgments being determined by a wholistic template comparison and "different" judgments resulting from a selfterminating serial process in which subjects appear to scan facial features sequentially from top to bottom. In contrast to such findings, Carey and Diamond (1977) indicate that by the age of 10 years children can successfully cope with recognition problems involving changes of expression, which they argue is suggestive of an ability to encode general facial configuration. This position was also taken earlier by Freedman and Haber (1974), who argued for a facial recognition process based upon the perceiver's construction of a coherently organized perception rather than an assemblage of independently processed features. However, the stimuli used in this study comprised a unique feature set which probably prevented subjects from employing previously learned feature encoding strategies; as such, the study supports the general notion that pictures containing features that are more easily organized are better recognized than pictures with no apparent coherence, rather than the specific hypothesis that faces are encoded on a configurational or Gestalt basis.

One consequence of the suggestion of a piecemeal feature processing strategy has been an attempt to 
define empirically the saliency of particular features for facial recognition. In assessing the general findings in this area, one must be somewhat cautious, since many studies have employed schematic faces whose feature components bear only cartoonlike resemblance to real features and other investigations have been concerned with asking questions about which facial features are salient in conveying expressions and emotions. Studies in fixation patterns of adults have suggested that moving parts of the face, such as eyes and mouth, have importance for conveying nonverbal communication and emotion (Argyle, 1967; Ekman, Friesen, \& Ellsworth, 1972; Izard, 1971). In the study of schematic faces, Friedman, Reed, and Carterette (1971) rank nose, eyes and forehead, and mouth in order of decreasing saliency, whereas McKelvie (1973) reports that eyebrows and mouth contribute more to the meaning of a face. In the case of Photofit constructions of faces, Ellis, Shepherd, and Davis (1975) report that for one of the faces studied performance was more accurate for forehead and eyes on a memory reconstruction test, while for another face accuracy was better for forehead and mouth; in both cases, subjects appeared to give priority to the upper part of the face in making their reconstructions. In recognition memory for photographs of real faces, McKelvie (1976) suggests that eyes seem to have greater saliency than the mouth in the memory representation of a face. While there is some divergence of opinion among developmental psychologists concerning the emergence of preferential scanning patterns of faces by infants, Maurer and Salapatek (1976) report that while infants in the first month of life tend to fixate only upon the periphery of an adult face, 2-month-old infants are more likely to fixate internal facial features, particularly the eyes. Finally, in a class of experiments involving masking of features by noise within critical spatial frequency bands, Harmon (1973) suggests that visual importance is assigned to low-frequency facial features such as overall head shape and gross hairline. In summary, ears and chin are the only features of the face that these studies agree upon as lacking in saliency for recognition, expressing emotion, or conveying meaning.

The objectives of the present study are to reinvestigate the issues of feature saliency and processing strategy in the scanning of representations of human faces employing some additional controls that have prevented previous studies from drawing unambiguous conclusions. First, faces are constructed using the Identikit technique, a procedure which results in compositions that are reasonably life-like and which permits close control over the specific feature content of each face constructed. Second, a "same". "different" RT paradigm is employed whereby subjects make judgments concerning the similarity of simultaneously presented pairs of faces; in this respect, the study concentrates upon the manner in which faces are scanned and encoded and attempts to minimize the contribution of memory. The technique of Bradshaw and Wallace (1971), which examines RT as a function of facial similarity, has been adopted with two important modifications: first, pairs rather than lists of faces are presented to subjects; second, specific changes to individual facial features are manipulated. Both of these manipulations permit the examination of the effects of changes in particular features directly; for example, in the condition where just one feature of a pair is changed and all possible single feature changes are investigated (in the present case, hairline, eyebrows, eyes, nose, mouth, and chin), an analysis of RTs to specific features changed permits inferences to be drawn concerning order of scanning of features. In addition, in feature change conditions involving simultaneous multiple combinations of features, a more direct estimate of whether specific features are processed simultaneously may be deduced. In particular, the contention of Bradshaw and Wallace (1971) that the reduction in magnitude of "different" RTs as a function of increasing feature dissimilarity in a pair of faces constitutes evidence for a serial self-terminating feature processing strategy may be more rigorously examined, in view of the fact that it is possible to produce such a function under conditions whereby one subset of facial features is scanned in serial manner while a second subset is scanned in parallel. If the features scanned in parallel are a relatively small subset of total number of features, then the relative proportions of the two subsets in any feature change condition will be the main determinant of the mean RT for that condition. One final advantage of the present design over that of Bradshaw and Wallace is that it permits error rates for specific feature changes to be determined and attempts to make provision for any confounding produced by speed/accuracy tradeoff across conditions by manipulating subjects' instructional set.

\section{EXPERIMENT 1}

\section{Method}

Composite faces were constructed from transparent overlays of features drawn from a police "Identikit"; the categories of features used were hair, eyebrows, eyes, nose, mouth, and chin. Examples of features were selected on the basis of being readily discriminable from each other. Since the selection of ears was somewhat limited, the same pair was used throughout. Twenty faces were constructed using completely different combinations of features, with the exception of eyes, where only light and dark colored examples were available; half of the faces were assigned light colored and half dark colored eyes, although the detail surrounding the eyeball was unique for each exemplar. The remaining stimulus set was constructed by changing one, two, four, five, or all six of the features of the basic 20 faces. In the one- 
feature-change condition, there were five examples of each of the single features changed. In the two-feature-change condition, there were three examples of each of the following feature-change combinations: hair-nose, hair-chin, hair-mouth, eyebrow-mouth, eyebrow-nose, eyebrow-eye, eye-chin, eye-mouth, nose-chin, and mouth-chin. In the four-feature-change condition, there were three examples of each of the following changes: eyebrow-eyenose-mouth, eyebrow-nose-mouth-chin, eyebrow-eye-nose-chin, eyebrow-eye-mouth-chin, hair-eyebrow-mouth-nose, haireyebrow-mouth-chin, hair-eyebrow-eye-chin, hair-eyebrow-nosechin, hair-eyebrow-eye-mouth, and hair-eye-nose-mouth. In the five-feature-change condition, there were five examples of each of the following feature changes: hair-eye-nose-mouth-chin, hair-eyebrow-eye-nose-chin, hair-eyebrow-nose-mouth-chin, hair-eyebrow-eye-nose-mouth, hair-eyebrow-eye-mouth-chin, and eyebrow-eye-nose-mouth-chin. There were 30 examples of all six feature changes.

Apparatus. The composite stimuli were all photographed and reproduced on matt photographic paper to a constant face height of $7.7 \mathrm{~cm}$, and were then mounted on either side of the centerline of a black card such that when pairs of cards were placed in Fields 2 and Blank of a Scientific Prototype GB three-channel tachistoscope two faces appeared to the viewer side by side in the middle of the field. The luminances of the two fields were set at a level of $59.2 \mathrm{~cd} / \mathrm{m}^{2}$, as measured on the white areas of the faces; the contrast of the faces was 0.8 .

Field 1 served as an adapting/fixation field and was uniformly illuminated to a luminance of $59.2 \mathrm{~cd} / \mathrm{m}^{2}$; it contained a small, dark central fixation spot.

Response latencies were recorded by a digital timer actuated by the onset of the field containing the faces and terminated by a verbal response of the subject through a voice-actuation relay.

Procedure. The subjects were required to make verbal judgments as to whether a pair of simultaneously presented faces were the same or different. One group of subjects was given instructions emphasizing accuracy; a second group, instructions emphasizing speed; and a third group, neutral instructions. Instructions were manipulated in this manner to permit speed/accuracy tradeoff functions to be determined for each of the stimulus conditions. The subjects were required to view the fixation spot binocularly and were given a verbal "ready" signal before each face pair appeared. A verbal decision by the subject stopped the timer and extinguished the faces. The fixation field was not illuminated during the time the faces were presented.

For each feature change condition, there were 30 trials together with 50 trials requiring a "same" response, yielding a trial block length of 200 presentations, with trials for each condition random. ized throughout the trial block. A second set of 56 training stimuli sampled from each of the conditions in the same ratio of "same" to "different" pairs was also constructed for use as training trials. The subjects were tested over a 4-day period; on the first 2 days, they were given practice on the training stimuli with the list presented three times on each day and were given feedback on the correctness of their responses. On each of the latter 2 days they were tested on the 200 test stimuli, preceded by 20 training trials.

Subjects. Thirty volunteer subjects were each paid $\$ 2 / \mathrm{h}$ for their participation in the experiment. Ten subjects were assigned randomly to each of the instruction conditions.

\section{Results}

Mean RT of correct responses and percent error of all 30 subjects for each of the stimulus conditions are indicated in Figure 1. Data have been collapsed across the variable of instructional set, since analysis of variance revealed that, although there was a significant main effect of instructional set $[F(2,27)=$
23.63, $\mathrm{p}<.01]$, there was no significant interaction with feature condition for either RT $[F(10,135)=$ $1.19]$ or error rate $[F(10,135)=1.92]$. Both $\mathrm{RT}$ $[F(5,135)=28.32, p<.001]$ and error rate $[F(5,135)$ $=17.93, \mathrm{p}<.001]$ were found to differ significantly as a function of feature change condition. There appears to be no overall tendency for a speed/ accuracy tradeoff across conditions.

In order to gain insight into the strategy by which faces are processed, it is necessary to break down this general function and examine the effects of specific configurational changes in detail.

"Same" responses. In assessing the implications of "same" judgments in terms of facial processing strategy, some degree of caution must be exercised, since trials requiring such judgments constituted only $25 \%$ of the total. Given this limitation, the question arises as to whether the longer decision time for "same" responses is consistent with a search strategy in which subjects check for differences among all the features, with response time determined by the slowest feature discrimination. An examination of Table 1, which indicates mean RT and percent error for "different" responses to specific feature changes, reveals that the slowest feature change discriminations take almost $.7 \mathrm{sec}$ longer, on average, than "same" decisions, contrary to the expectations of a simple exhaustive feature scan model. The alternate position that "same" judgments are based upon a wholistic or template comparison is consistent with the finding that "same" responses are faster than the slowest "different" response to a specific feature

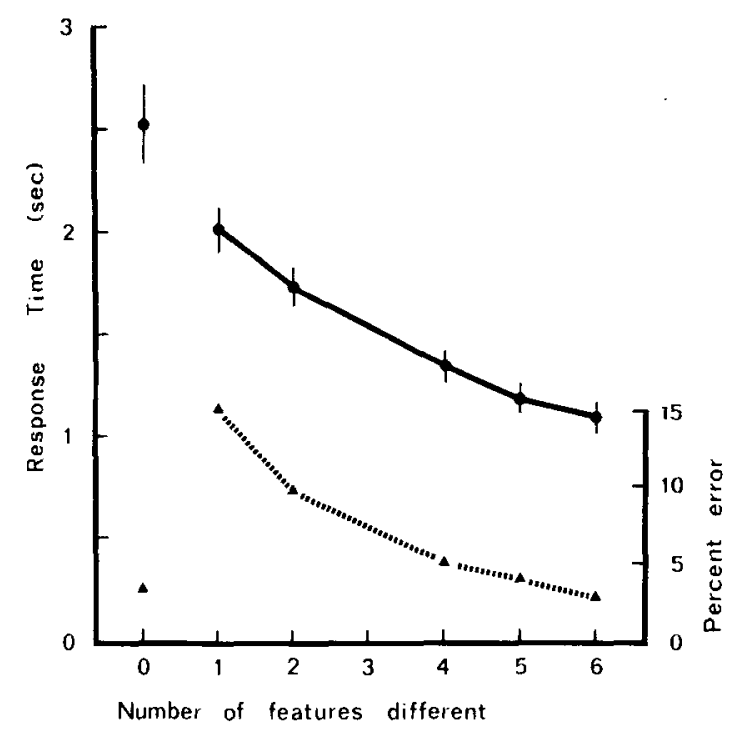

Figure 1. Mean RT (solid line) and mean percent error (broken line) as a function of number of features different in a pair of faces. Variability is indicated by one standard error either side of mean. 
Table 1

Mean Reaction Time (RT) and Mean Percent Error (E) for Each Particular Feature Change for One- (1FC), Two- (2FC), Four- (4FC), and Five- (5FC) Feature Change Conditions

\begin{tabular}{|c|c|c|c|c|c|c|c|c|c|c|c|}
\hline \multicolumn{3}{|c|}{$1 \mathrm{FC}$} & \multicolumn{3}{|c|}{$2 \mathrm{FC}$} & \multicolumn{3}{|c|}{$4 \mathrm{FC}$} & \multicolumn{3}{|c|}{$5 \mathrm{FC}$} \\
\hline & $\mathrm{RT}$ & E & & RT & E & & RT & $\mathrm{E}$ & & RT & $E$ \\
\hline $\mathrm{H}$ & 1.450 & 7.3 & $\mathrm{HC}$ & 1.195 & 1.7 & HEbEC & 1.060 & 0.0 & HEbEMC & 1.067 & 3.3 \\
\hline $\mathrm{C}$ & 1.487 & 8.7 & EC & 1.222 & 6.7 & HEbNC & 1.208 & 0.0 & HENMC & 1.106 & 6.7 \\
\hline $\mathrm{E}$ & 1.507 & 6.0 & $\mathrm{HN}$ & 1.341 & 6.7 & HEbEM & 1.210 & 0.0 & HEbENC & 1.172 & 3.3 \\
\hline $\mathrm{Eb}$ & 2.248 & 16.7 & $\mathrm{HM}$ & 1.441 & 5.0 & HENM & 1.216 & 0.0 & HEbNMC & 1.227 & 3.3 \\
\hline $\mathrm{N}$ & 3.09 & 28.3 & $\mathrm{EM}$ & 1.494 & 4.5 & HEbMC & 1.244 & 8.3 & HEbENM & 1.230 & 0.0 \\
\hline \multirow{5}{*}{ M } & 3.16 & 24.7 & $\mathrm{CN}$ & 1.507 & 10.0 & EbENC & 1.263 & 6.7 & EbENMC & 1.302 & 6.7 \\
\hline & & & $\mathrm{EEb}$ & 1.511 & 8.4 & EbEMC & 1.265 & 6.7 & & & \\
\hline & & & $\mathrm{CM}$ & 1.526 & 13.4 & EbNMC & 1.548 & 10.0 & & & \\
\hline & & & EbM & 2.43 & 16.7 & EbENM & 1.575 & 6.7 & & & \\
\hline & & & NM & 3.790 & 21.7 & HEbMN & 1.614 & 13.3 & & & \\
\hline
\end{tabular}

Note-Italicized reaction time means are not significantly different $(p<.01)$ on a Newman - Keuls test. $H=$ hair; $C=$ chin; $E=$ eyes; $E b=$ eyebrow; $N=$ nose $M=$ mouth.

change. A further explanation has been suggested by Hawkins (1969), who proposed that under some conditions subjects may terminate prematurely their search through features and make a "same" response; this incomplete analysis of all features is likely to occur when the subjects perform discriminations on multiattribute stimuli when more than one attribute is important. It is suggested that this behavior is evidenced by high error rates when such stimuli differ with respect to only the least discriminable attribute. The high error rates in the one feature change condition for changes to either nose or mouth (the longest RTs) are consistent with this interpretation. Further support for such an explanation is provided by an analysis of false "same" responses for this condition, for which the overall mean RT was $2.35 \mathrm{sec}$ compared with the mean of $2.56 \mathrm{sec}$ for correct "same" responses in the zero feature change condition. The lower mean for incorrect compared with correct "same" responses is to be expected according to Hawkins, since the former is the result of trials in which the subject always prematurely terminates, whereas the mean for correct "same" responses is based upon occasions not only when the subject occasionally prematurely terminates but also trials in which processing continues until all features are processed.

"Different" responses. Mean RT for correct responses and error rates for the four conditions that permit examination of "different" responses to specific configurational changes in features are indicated in Table 1. In the 1FC condition, there are clear differences in both RT $[F(5,145)=33.99$, $\mathrm{p}<.001]$ and error rate $[\mathrm{F}(5,145)=17.93, \mathrm{p}<.001]$ between individual features; the same positive relationship between mean RT and error rate found in the overall function is again observed within this feature change condition. Differences between RTs for different facial features were explored more closely, using Newman-Keuls, with the outcome indi- cated in Table 1. Although 25 subjects reported a serial scan of faces from top to bottom, there is no tendency for the data to follow the expected ordering for specific feature changes. The similarity of means for changes involving hairline, eye, and chin, although open to other interpretations (which are pursued in the discussion) is consistent with the hypothesis that these three features are scanned in parallel. The veracity of this hypothesis may be assessed by examining other feature change conditions for evidence of similar RTs to the $1 \mathrm{FC}$ condition whenever one of the three features in question is changed irrespective of any accompanying changes among the remaining feature set.

In the $2 \mathrm{FC}$ condition, there is again a significant effect of specific feature changes on both RT $[F(9,261)=45.64, p<.001]$ and error rate $[F(9,261)$ $=20.99, \mathrm{p}<.001]$; closer inspection of individual means reveals a tendency for feature changes that involve hairline, eyes, and chin to be faster than pairs involving eyebrows, nose, and mouth. If a comparison is made between means in the $2 \mathrm{FC}$ condition involving hairline, eyes, and chin (excluding changes where they appear in combination with each other) with means for the same features in the 1FC condition, a high degree of similarity is observed; this similarity will be examined more closely below. A simultaneous change involving any two of this subset of features in combination produces faster RTs than to either any single one (compared with the 1FC condition) or to any one in combination with eyebrows, nose, or mouth.

In the 4FC condition, a significant effect of specific feature changes on both $\operatorname{RT}[F(9,261)=3.48, p<.01]$ and error rate $[F(9,261)=3.14, p<.01]$ is again obtained. The feature change conditions containing just one example of hairline, eyes, or chin again produce RTs similar to comparable feature manipulations in the $1 \mathrm{FC}$ and $2 \mathrm{FC}$ conditions. There is further evidence that when these features appear in com- 
binations, RT decreases as a function of the number combined; this trend may be seen again in the 5FC condition, although there is no overall effect of specific feature changes on either $\operatorname{RT}[F(5,145)=2.13]$ or error rate $[F(5,145)=2.06]$.

A comparison of feature changes involving hairline, eyes, and chin as single, double, and threefold combinations across the four-feature-change conditions is made in Table 2. The similarity in mean RTs involving changes in one of these features across the three feature changes is supported by a NewmanKeuls test which indicates that none of the differences between the relevant comparisons reaches significance $(\mathrm{R} 2=.351, \mathrm{R} 3=.421, \mathrm{p}=.05)$. Comparisons involving twofold and threefold combinations of these features indicate a marked similarity in RT across feature-change conditions. There appears to be a consistent trend for RT to decrease as a function of the number of these features combined together within a feature change.

Although the data for the other facial features are not as extensive, it does not appear as if they have any systematic influence on RT when combined with hairline, eyes, or chin. In contrast to the latter, RTs from feature changes involving eyebrows, nose, and mouth either as single or combined changes are characterized by high error rates and long RTs. In addition, there is no tendency for RT to be determined by the faster feature of a pair when changed in a twofold combination or for RT to be lower in such circumstances than for the $1 \mathrm{FC}$ condition.

\section{Discussion}

The general difficulty in determining evidence for serial-parallel distinctions in RT data has been well documented (Egeth, 1966; Nickerson, 1972; Townsend, 1971). In the present experiment, a further complexity arises out of the fact that the overall response time to a specific feature change is a reflection of not only the mode of processing facial features, but also of the degree of difficulty involved in making discriminations among specific features. In many previous experiments involving "same"'-"different" judgments with multiattribute stimuli, the attributes have been readily discriminable (size, color, shape), whereas in the present case, differences between features along a feature dimension may be much more subtle. It may be argued that, with the Identikit features used in the present experiment, the consistently higher error rates for discriminations involving eyebrows, nose, and mouth can be taken as evidence that changes in these features are less discriminable than in other facial features. In order to assess the magnitude of the possible confound played by differential feature discriminability in the previous experiment, a second experiment was carried out in which response latency was measured for discriminations
Table 2

Mean Reaction Times Across Feature Change Conditions for Single, Twofold, and Threefold Combinations in Hairline (H), Eyes (E), and Chin (C)

\begin{tabular}{|c|c|c|c|c|c|}
\hline & $1 \mathrm{FC}$ & $2 \mathrm{FC}$ & $4 \mathrm{FC}$ & $5 \mathrm{FC}$ & Mean \\
\hline \multicolumn{6}{|c|}{ Single } \\
\hline $\mathrm{H}$ & 1.450 & 1.391 & 1.614 & & \\
\hline $\mathrm{E}$ & 1.507 & 1.503 & 1.575 & & \\
\hline $\mathrm{C}$ & 1.487 & 1.517 & 1.548 & & \\
\hline Mean & 1.481 & 1.471 & 1.579 & & 1.51 \\
\hline \multicolumn{6}{|c|}{ Twofold } \\
\hline $\mathrm{HC}$ & & 1.195 & 1.244 & 1.227 & \\
\hline HE & & & 1.213 & 1.230 & \\
\hline $\mathrm{EC}$ & & 1.222 & 1.264 & 1.302 & \\
\hline Mean & & 1.209 & 1.240 & 1.253 & 1.234 \\
\hline \multicolumn{6}{|c|}{ Threefold } \\
\hline HEC & & & 1.060 & 1.115 & 1.101 \\
\hline
\end{tabular}

between pairs of features presented without a facial context.

\section{EXPERIMENT 2}

\section{Method}

The six exemplars of each feature used in the previous experiment in the construction of faces were photographed and reproduced in isolation to the same scale as in Experiment 1. Each was mounted and presented in a similar manner, with each feature occurring in its correct facial position relative to the fixation mark. The feature pair again appeared adjacent to each other on either side of the centerline, separated by the same amount as in Experiment 1 . The fields used and luminances were also similar to those used in the first experiment.

Procedure. The general procedure employed in the first experiment was repeated, except that a condition of neutral instructional set was adopted. For each feature condition, each exemplar was paired with every other exemplar and with itself, to give a total of 21 pairs, 15 of which required a "different" response, and 6 a "same" response, thus preserving approximately the equivalent ratio of "same" to "different" trials as the first experiment. Total trial block length was 126 trials, with features and exemplars randomized throughout the trial block. Subjects were tested over a 3-day period, with the list presented in a different randomized sequence of trials each day, preceded by 20 practice trials using different feature exemplars. Ten new volunteer subjects were paid $\$ 3 / \mathrm{h}$ for their participation.

\section{Results}

Mean response latencies and error rates summed across trials, exemplars and subjects are indicated for "same" and "different" comparisons in Table 3. Also shown is the range of response latencies between means of the fastest and slowest exemplars of each feature. Analysis of variance of "different" response latencies produced a significant effect of feature type $[F(5,45)=6.10, p<.001]$ and trials $[\mathrm{F}(5,45)=13.21, \mathrm{p}<.001]$ but a nonsignificant interaction between these two factors $[F(25,225)=$ 1.37]. The trials effect was due to a systematic decrease in response latencies throughout the experiment from a mean of $.898 \mathrm{msec}$ on the first trial block to a mean of $.793 \mathrm{msec}$ on the sixth. 
Table 3

Mean Reaction Time (in Seconds) and Percent Error Summed Over Subjects and Exemplars for "Same" and "Different" Judgments for Different Facial Features

\begin{tabular}{|c|c|c|c|c|c|}
\hline \multirow[b]{2}{*}{ Feature } & \multicolumn{2}{|c|}{$\begin{array}{c}\text { "Same" } \\
\text { Judgments }\end{array}$} & \multicolumn{2}{|c|}{$\begin{array}{l}\text { "Different" } \\
\text { Judgments }\end{array}$} & \multirow[b]{2}{*}{ Range } \\
\hline & RT & $\begin{array}{c}\text { Percent } \\
\text { Error }\end{array}$ & RT & $\begin{array}{l}\text { Percent } \\
\text { Error }\end{array}$ & \\
\hline Hairline & .893 & .37 & .783 & 0 & .164 \\
\hline Eyebrows & .918 & 2.22 & .891 & 2.78 & .221 \\
\hline Eyes & .924 & 1.85 & .890 & 2.96 & .140 \\
\hline Nose & .975 & 1.48 & .846 & .56 & .186 \\
\hline Mouth & .925 & 0 & .856 & .74 & .157 \\
\hline Chin & .914 & 1.30 & .859 & .46 & .212 \\
\hline
\end{tabular}

Note-RT range between most and least discriminable exemplar pair for each feature.

The differences between features were examined with a Newman-Keuls test which indicated that the only significant difference was between the mean for "hair" and all other features, ( $\mathrm{df}=45, \mathrm{p}<.01)$. Latencies of "same" responses showed a similar pattern of relative magnitude between different features as "different" responses but were on average longer by about $63 \mathrm{msec}$.

\section{DISCUSSION}

The finding of nonequal discriminability between different Identikit facial features raises questions concerning the validity of the comparisons between feature change conditions that were made on the basis of the results of the first experiment. However, there are several comparisons that may be performed with the data from the discrimination experiment to determine whether the differences in means obtained in the first experiment were due to processing strategy or an artifact of differential feature discriminability. Table 4 shows mean response latencies for discrimination of single pairs of features in Experiment 2 for those features previously employed in the whole-face condition for that particular feature comparison. For each feature-change condition, the mean is summed across all such exemplars for that condition, and for multiple-feature-change conditions, the mean is summed over all feature pairings. In addition, the most discriminable features for each condition are indicated in parentheses with their associated response latencies.

If it is assumed that either the overall discriminability of exemplars of a particular feature or the most easily discriminable exemplar (in the case of multiple feature changes) determines response latency in the case when subjects are required to make a discrimination between such exemplars in the context of a face, then a strong positive correlation between mean response latencies for each of the featurechange conditions of the first experiment and mean latencies for the same exemplars in Experiment 2 should be obtained. These correlations for the fourfeature-change conditions, respectively, were -.152 , $-.205, .167$, and - .086; similar correlations between the most discriminable feature in the multiplefeature-change conditions and response latencies from Experiment 1 were $-.511, .088, .207$, and - .016. These correlations provide little support, therefore, for the hypothesis that the ordering of response latencies in Experiment 1 was the result of discriminability differences in the exemplars used.

A second check on the possible confounding effect of differential discriminability of feature exemplars may be obtained for the 1FC condition by subtracting the latency for an exemplar compared in isolation in Experiment 2 from the latency obtained for the same exemplar in the full-face context of Experiment 1 . This yields the following mean durations in seconds: eyes, .595; chin, .613; hairline, .656; eyebrows, 1.362; nose, 2.268; and mouth, 2.297. When

Table 4

Mean Reaction Time (in Seconds) for Discriminability of Isolated Features Based on the Exemplars Used for Whole Faces in Experiment 1 for One- (1FC), Two- (2FC), Four- (4FC), and Five- (5FC) Feature Change Conditions

\begin{tabular}{|c|c|c|c|c|c|c|c|c|c|c|c|}
\hline \multicolumn{3}{|c|}{$1 F C$} & \multicolumn{3}{|c|}{$2 \mathrm{FC}$} & \multicolumn{3}{|c|}{$4 \mathrm{FC}$} & \multicolumn{3}{|c|}{$5 \mathrm{FC}$} \\
\hline & Mean & Fastest & & Mean & Fastest & & Mean & Fastest & & Mean & Fastest \\
\hline $\mathrm{H}$ & .783 & .733 & $\mathrm{HC}$ & .799 & $.728(\mathrm{H})$ & HEbEC & .852 & $.793(\mathrm{C})$ & HEbEMC & .906 & $.805(\mathrm{~Eb})$ \\
\hline $\mathrm{C}$ & .859 & .827 & EC & .887 & .847 (C) & $\mathrm{HEbNC}$ & .870 & $.717(\mathrm{H})$ & HENMC & .851 & .779 (H) \\
\hline $\mathrm{E}$ & .89 & .807 & HN & .820 & $.733(\mathrm{H})$ & HEbEM & .872 & $.798(\mathrm{H})$ & HEbENC & .856 & $.732(\mathrm{~N})$ \\
\hline $\mathrm{N}$ & .846 & .738 & HM & .896 & $.861(\mathrm{M})$ & HENM & .870 & $.701(\mathrm{~N})$ & HEbNMC & .849 & $.701(\mathrm{~Eb})$ \\
\hline M & .856 & .764 & EM & .922 & $.910(\mathrm{E})$ & $\mathrm{HEbMC}$ & .777 & .717 (C) & HEbENM & .843 & $.722(\mathrm{~N})$ \\
\hline \multirow[t]{5}{*}{$\mathrm{Eb}$} & .891 & .729 & $\mathrm{CN}$ & .890 & $.739(\mathrm{~N})$ & EbENC & .912 & .749 (E) & EbENMC & .901 & $.778(\mathrm{M})$ \\
\hline & & & EEb & .845 & $.763(\mathrm{~Eb})$ & EbEMC & .801 & $.757(\mathrm{E})$ & & & \\
\hline & & & $\mathrm{CM}$ & .849 & $.835(\mathrm{C})$ & EbNMC & .857 & $.808(\mathrm{~N})$ & & & \\
\hline & & & EbM & .816 & $.805(\mathrm{~Eb})$ & EbENM & .875 & $.794(\mathrm{~Eb})$ & & & \\
\hline & & & NM & .842 & .759 (M) & HEbMN & .887 & $.759(\mathrm{M})$ & & & \\
\hline
\end{tabular}

Note-Means for multiple feature change conditions are summed across exemplars of all features within a particular grouping. Reaction time to the most discriminable feature exemplars within each category is indicated together with feature type in parentheses. $H=$ hair $C=$ chin; $E=$ eyes; $N=$ nose; $M=$ mouth; $E b=$ eyebrows. 
these means are subjected to a Newman-Keuls analysis using the same error term as for the first experiment, an identical clustering of means to that found previously is obtained.

As a final check on the role played by feature discriminability, mean discriminability latencies were calculated for the comparisons performed previously in Table 2 involving the subset of hairline, eyes, and chin. Latencies for the exemplars used for these comparisons have been calculated from the data on isolated feature comparisons in Experiment 2 and are presented in Table 5 . It can clearly be seen that the tendency for the means to decrease as a function of the number of combined features which was obtained in the first experiment is not due to changes in discriminability of the particular feature exemplars that were employed.

Thus, the overall implications of Experiment 2 are that, while there are significant differences in discriminability between the different facial features drawn from the Identikit and also differences between exemplars within a particular feature, there is no evidence to suggest that such differences either account for or confound the results of the first experiment. The response latency data obtained in Experiment 2 confirm the differential discriminability of features reported previously by Davies, Ellis, and Shepherd (1977) based upon error rates obtained from comparisons of faces constructed from the "Photofit" system used in Britain. However, the relative ordering of feature discriminability (from most to least) in their study was forehead, eyes, mouth, chin, and nose compared with hairline, nose, mouth, chin, eyes, and eyebrows in the present study. Differences in the physical characteristics of the stimuli (e.g., size, contrast) and also the task demands in the two studies may account for this discrepancy.

To return to the implications of the data from Experiment 1 for processing strategy, let us consider four self-terminating processing models that have been suggested for tasks similar to the present: (1) serial/random order of interrogation, (2) serial/ nonrandom order of interrogation, (3) parallel/ variable decision time, and (4) parallel/continual accumulation over features. The first model was selected by Bradshaw and Wallace (1971) to account for data obtained in an experiment that employed essentially the same paradigm as the present. According to the model, features are checked serially in a random order and processing is terminated as soon as the first distinguishing critical feature is encountered. Even though close replication of their function describing the relationship between RT and number of features different has been obtained, detailed examination of the RTs to specific feature change reveals no support for sequential feature by feature analysis. The second serial model which proposes
Table 5

Mean Reaction Times for Discriminability of Isolated Features Across Feature Change Conditions for the Exemplars Used for Whole Faces in Experiment 1 for Single, Twofold, and Threefold Combinations of Changes in Hairline (H), Eyes (E), and Chin (C)

\begin{tabular}{lccccc}
\hline & $1 F C$ & $2 F C$ & $4 F C$ & $5 F C$ & Mean \\
\hline H & .794 & .774 & .785 & & \\
E & .912 & .918 & .888 & & \\
C & .874 & .901 & .889 & & .859 \\
Mean & .860 & .864 & .854 & \\
\multicolumn{5}{c}{ Twofold } \\
HC & \multicolumn{5}{c}{ Single } \\
HE & \multicolumn{7}{c}{.799} & .755 & .887 & \\
EC & \multicolumn{7}{c}{.887} & .827 & .920 & \\
Mean & \multicolumn{7}{c}{.843} & .810 & .891 & .848 \\
HEC & \multicolumn{5}{c}{ Threefold } \\
\hline
\end{tabular}

Note-Means are summed across features in multiple feature change conditions.

that subjects do not select randomly from all features but first select at random from the subset of hairline, eyes, and chin, and then select eyebrows, and finally choose at random from nose and mouth would account for a large proportion of the data. This model would explain the consistent decrease in response latencies obtained with multiple feature changes for the subset hairline, eyes, and chin (as indicated in Table 2) in terms of the increased probability of encountering a feature for which a mismatch can be obtained as a function of the number of features that differ between the two faces. The third and fourth models differ from the second in that it is assumed that features of the subset hairline, eyes, and chin are first processed in parallel, followed by a slower serial comparison of the remaining features. The latter dual-processing strategy has been proposed by Neisser (1967) and has received some limited empirical support by Beller (1970) as a result of experiments involving "same"-"different" judgments with letter strings. In order to determine whether the present data can support such a model, it is necessary to reexamine the changes in RT that occur when combinations of features are simultaneously changed. Although, in general, RT is determined by features of the subset hairline, eyes, and nose, when combined with any of the remaining subset RT is faster when two of the former subset are combined together and faster again when three are combined. These trends are consistent with findings of Hawkins (1969), and Nickerson (1967) who demonstrated that "different" RTs declined as a function of the number of different attributes of a stimulus and that "composite" RTs were less than the least of the relevant "component" RTs. 
In detail, Model 3 attempts to account for these trends in the data by postulating an overlap in decision time distributions of each feature discrimination channel, such that at the time of making a decision the more channels that signal "different," the faster a decision may be made. The data show that the RT to a feature difference when only one feature is different is reduced by as much as $\mathbf{4 0 0} \mathrm{msec}$ when three features are different, which implies a minimum decision time of at least $400 \mathrm{msec}$ for the condition in which only one feature differs. This model therefore yields an estimate of decision time which is considerably longer than total RT in previously reported tasks involving a feature comparison process. A better account of the reduction in RT with multiple-feature changes involving hairline, eyes, and chin is given by Model 4, which assumes that each of these features is compared in parallel and that within each feature channel the comparison process yields an output which is a graded indication of the degree of the mismatch obtained. A decision is based upon the aggregation of mismatch information over multiplefeature channels. ${ }^{1}$

A major difficulty for each of the models is posed by the data from feature change conditions involving eyebrows, nose, and mouth, since this subset does not show the pattern of decreased RTs when features within this set are combined simultaneously. An examination of the mean RTs shows that they are ordered as eyebrows fastest followed by nose and mouth; inspection of the ordering for individual subjects reveals that 23 show this pattern, and hence it is not an averaging artifact. When two features of this set are combined, although the present data are somewhat limited, there is no indication that RT is determined by the faster of the two components. An examination of the two-feature combinations (eyebrow/mouth and nose/mouth) does not provide any insights into processing strategy; in the former case, the RT for the two features changed together is approximately the mean of the two component features when changed in isolation; in the latter case, RT is slower in the combined condithan the individual components. The limited evidence available that features in the subset of eyebrows, nose, and mouth do not produce similar patterns of RT when combined compared with hairline, eyes, and chin, and the fact that features of the former subset when compared with the latter subset fail to produce further reductions in $\mathrm{RT}$, is consistent with the hypothesis that different processing strategies are involved for the two subsets of features.

Given the general difficulty in attempting to distinguish between serial and parallel processing models and the fact that none of the above can give an entirely satisfactory account of the effects of all of the feature change conditions, it is perhaps necessary to examine the general strategy that a subject might adopt in order to understand why some features (hairline, eyes, and chin) are given apparent processing priority.

It is evident from questioning subjects after the experiment that multiple eye movements from one face to the other are made in this task; this would account for the relatively long latencies obtained in the present study compared with others mentioned previously. A recently reported study of the eyemovement patterns of subjects performing a simultaneous comparison on pairs of faces suggests that the same facial feature must be fixated in each face before a decision can be made (Walker-Smith, Gale, and Findlay, 1977) and, in addition, that subjects normally show two or more fixations from one face to the other. It is also suggested that subjects pay particular attention to the internal features of a face in order to integrate features into a general facial gestalt. In applying these findings to the present study, it is proposed that the strategy adopted by subjects in Experiment 1 was to make a rapid glance in the general direction of the eyes first to one face and then to the other; the relatively high spatial frequency information concerning eyes would then be centered upon the fovea and low-frequency information from the hairline and chin which describe overall face shape could be extracted from the periphery. The overall impression of the face obtained from these features in the first fixation would then be compared with the second fixation, possibly by means of a serial/random order or parallel/cumulation over features process as outlined above. If the subject fails to find a mismatch, internal features of the face are scanned sequentially from top to bottom with repeated glances between faces. Using an estimate of fixation duration and movement time of $300 \mathrm{msec}$ (Yarbus, 1967), there is clearly time for subjects to make one fixation for each stimulus of the feature pair in the discrimination experiment; in the face comparison situation, the fastest response latencies suggest that no more than three fixations can be made. The validity of these assumptions concerning the role of eye movements in both the feature and facial discrimination tasks is currently being evaluated in a series of experiments in which eye movements are monitored by means of a corneal reflection technique.

Finally, in attempting to generalize the implications of a dual-processing strategy suggested by the above data to the wider problem of everyday facial recognition, a major limitation is imposed by the nature of the stimuli. Identikit representations may have little ecological validity, since the feature set is somewhat stylized and limited in range compared with real features, and, additionally, such representations, like photographs, may inhibit utilization of 
previously learned scanning patterns in which greater attention is allocated to the more mobile facial features. With respect to the issue of saliency of features, both RTs and error rates tend to implicate the hairline, eyes, and chin as being the most salient in this situation, and, although this may be a taskdependent effect, the finding is consistent with previous studies on feature saliency in recognition memory and bears a striking similarity to scanning and feature preferences of early infant behavior.

\section{REFERENCES}

ARGYLe, M. The psychology of interpersonal behaviour. London: Cox and Wyman, 1967.

Beller, H. K. Parallel and serial stages in matching. Journal of Experimental Psychology, 1970, 84, 213-219.

Bradshaw, J. L., \& Wallace, G. Models for the processing and identification of faces. Perception \& Psychophysics, $1971,9,443-448$.

Carey, S., \& Diamond, R. From piecemeal to configurational representations of faces. Science, 1977, 195, 312-313.

Davies, G., Ellis, H., \& ShePherd, J. Cue saliency in faces as assessed by the 'Photofit' technique. Perception, 1977, 6, 263-269.

Egeth, H. E. Parallel versus serial processes in multidimensional stimulus discrimination. Perception \& Psychophysics, $1966,1,245-252$.

Ekman, P., Friesen, W. F., \& Ellsworth, P. Emotion in the human face. New York: Pergamon Press, 1972.

Ellis, H., Shepherd, J., \& Davies, F. An investigation of the use of Photo-fit technique for recalling faces. British Journal of Psychology, 1975, 66, 29-37.

Freedman, J., \& Haber, R. N. One reason why we rarely forget a face. Bulletin of the Psychonomic Society, 1974, 3, 107-109.

Friedman, M. P., Reed, S. K., \& Carterette, E. C. Feature saliency and recognition memory for schematic faces. Perception \& Psychophysics, 1971, 10, 47-50.

Harmon, L. D. The recognition of faces. Scientific American, $1973,229,71-82$.
Hawkins, H. L. Parallel processing in complex visual discrimination. Perception \& Psychophysics, 1969, 5, 56-64.

IzARD, C. E. The face of emotion. New York: Appleton-CenturyCrofts, 1971.

MAURER, D., \& SAlapatex, P. Developmental changes in the scanning of faces by young infants. Child Development, 1976, 47, $523-527$

McKelvie, S. J. The meaningfulness and meaning of schematic faces. Perception \& Psychophysics, 1973, 14, 343-348.

McKelvie, S. J. The role of eyes and mouth in the memory of a face. American Joumal of Psychology, 1976, 89, 311-323.

Neisser, U. Cognitive psychology. New York: Appleton-CenturyCrofts, 1967.

Nickerson, R. S. "Same"-"different" response times with multi-attribute stimulus differences. Perceptual and Motor Skills, 1967, 24, 543-554.

Nickerson, R. S. Binary-classification time: A review of some studies of human information-processing capabilities. Psychonomic Monograph Supplements, 1972, 4, 275-318.

SMITH, E. E., \& NiELSEN, G. D. Representations and retrieval processes in short-term memory: Recognition and recall of faces. Journal of Experimental Psychology, 1970, 85, 397-405.

Townsend, J. T. A note on the identifiability of parallel and serial processes. Perception \& Psychophysics, 1971, 10, 161-163.

TVERSKy, B. Pictorial and verbal encoding in a short-term memory task. Perception \& Psychophysics, 1969, 6, 225-233.

Walker-Smith, G. J., Gale, A. G., \& Findlay, J. M. Eye movement strategies involved in face perception. Perception, $1977,6,313-326$.

Yarbus, A. L. Eye movements and vision. New York: Plenum Press, 1967.

YIN, R. K. Looking at upside-down faces. Journal of Experimental Psychology, 1969, 81, 141-145.

\section{NOTE}

1. I should like to thank an anonymous reviewer for several critical comments and helpful suggestions concerning implications of the processing models and for proposing explicitly Model 4, which I had not previously considered.

(Received for publication May 24, 1977; revision accepted November 8, 1977.) 